\title{
Synchronous Control of Linear Servo Systems for CNC Machine Tools
}

\author{
Mi-Ching Tsai*, Min-Fu Hsieh and Wu-Sung Yao \\ Department of Mechanical Engineering \\ National Cheng Kung University \\ Tainan 701, Taiwan, ROC \\ *E-mail: mctsai@mail.ncku.edu.tw \\ Tel: +886-6-2352344 \\ Fax: $+886-6-2352973$
}

Keywords: multi-axis, synchronous control, linear servomotor, master/slave control, repetitive control

\begin{abstract}
This paper presents a synchronous control scheme for a high-speed machine tool using linear servomotors. The gantry machine tool is mainly composed of three linear servomotors: one for the $\mathrm{X}$-axis and the other two as a parallel pair for the Y-axis. The advantage of such a structure with two parallel linear servomotors for a single axis is to enable the machine to operate at high speed and acceleration and to increase the stiffness and precision. However, the major concern is the precisely synchronous movement of the pair of linear servomotors, and to overcome this problem, a master/slave control technique is applied. Repetitive control for periodic errors compensation is also considered here. Experimental results are presented to demonstrate the effectiveness of the proposed control systems.
\end{abstract}

\section{Introduction}

The capability for multi-axial machine tool feed drives to follow specified trajectories accurately is an important requirement for precision machining, especially in view of the increasing interest in high-speed machining of linear motors feed drives. Control schemes that are capable of reducing contour errors are particularly important for this application since such error component is directly related to the accuracy of machined parts [12]. The coordination of multiple feed drive axes is achieved in machine tools by coordinating the commands to the multiple feed drives. It is assumed implicitly by such an approach that the individual feed drive servomechanisms ensure accurate tracking of the axis command inputs. This assumption is valid at low contouring speeds but not so at higher speeds. The control algorithms commonly employed may result in nonzero following errors, which increase as the contouring speed increases.

Many automation applications that incorporate an isolated motion component, i.e., an individual thrust cylinder, rotary actuator, or spindle, are adequately addressed using dedicated single-axis controllers. In industrial applications, individual axis control strategy is adopted in most of the multi-axis motion control problems [12]. To reduce contour errors, indirect approach by increasing the position tracking accuracy of individual axis is commonly used. Many algorithms have been proposed to fulfill these demands, for example, feed-forward control [12] [15], preview control [1], and zero phase error tracking control [3]. However, multi-axis control capability may provide significant economy for commanding the operations of numerous independent motion axes, and it is essential for coordinating the motion of elements in complex materials-working and spatial-measurement devices.

Synchronous control [7] [11] [12] [15] provides a unique set of advantages and opportunities to error compensation of the corresponding multi-axis motion control. A feasible algorithm to achieve a high accuracy of multi-axis motion control is cross-coupled control [2] [5-6] [15]. The majority of the previous works on cross-coupling control was proposed for machine tools and robotics [2] [11]. In cross-coupled control, the entire multi-axis system is considered as a single system. Compensations are calculated by taking into consideration of the mutual influences among axes to increase the degree of matching among axes and consequently reduce the tracking error. Another synchronous control scheme is called the master/slave control technology [10], where for a biaxial system the reference input for the slave axis is generated based on the measured master axis position and the desired contour. A simplified master/slave system is shown in Fig. 1, in which the follower unit accurately tracks the master at some velocity or positional ratio to provide the motion control equivalents of a gearbox and a cam mechanism. High-speed on-the-fly cutting can be implemented by slaving the tool positioners to the material transporter. Other applications include conveyor-line synchronization, coil winding, and machining spindle control.

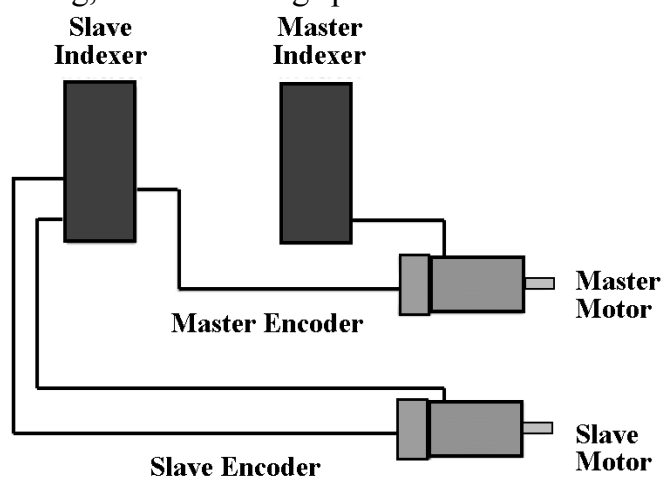

Fig. 1 The master/slave control configuration

Nowadays, many kinds of servo systems have been utilized in production and assembly lines. Linear motors, with their non-contacting energy translation and high acceleration essences, have been increasingly applied in high-speed and high-accuracy industries such as IC manufacturing equipment and machining tools.

Therefore, this paper presents a control algorithm directed specifically for the linear servo system of a 
high-speed gantry machine tool. The machine tool is composed of a pair of parallel linear servomotors for the $\mathrm{Y}$-axis and a linear motor with a beam striding across the pair of parallel linear servomotors for the $\mathrm{X}$-axis, as shown in Fig. 2. For such a case, controlling the pair of parallel linear servomotors to achieve precisely synchronous movement at high speed is the major challenge for the present study. Note that the two parallel linear motors only form a single axis. Rather than contour errors for a biaxial system, the error between the two parallel motors is called the "synchronization error". Hence, the proposed synchronous control technique based on the master/slave control aims at zero synchronization errors for the two parallel linear servomotors. Periodic signal input is also considered, where the repetitive controller compensates the periodic error. Experiments are also conducted on the above-mentioned machine tool.

This paper is organized as follows. In Section 2, the mathematic model for the parallel linear servo system is derived. Section 3 discusses the design of the synchronous control scheme. The experimental studies are presented in Section 4, followed by Section 5, which closes the paper with brief conclusions.

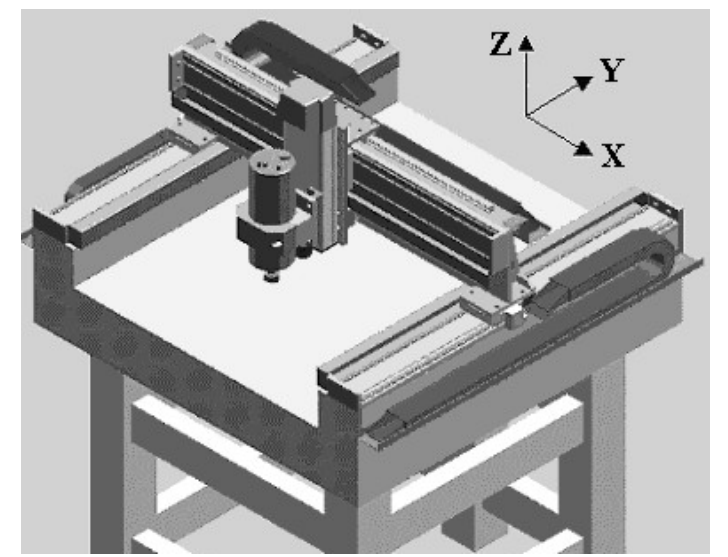

Fig. 2 Configuration of the linear servomotor driven machine tool

\section{Modeling of the Linear Servo System}

As previously mentioned, the master/slave control technique is adopted to achieve zero synchronization error between the parallel linear motors. In the proposed master/slave control technique, position control is applied to one linear motor (master) and velocity control for the other (slave). The force resulted from the deformation of the X-axis components (i.e. a linear motor and the saddle) is compensated, if there is any non-synchronization between the two parallel motors. This force is simplified to be approximately proportional to the synchronization error, and the follower (slave) units accurately track the master at some velocity or positional ratio to eliminate the force and hence achieve zero synchronization error.

The kinematics of the parallel linear servomotor system with $\mathrm{X}$-axis (composed of a saddle and a linear motor) are analyzed, as illustrated in Fig. 3(a)(b)(c). The mass/material characteristics of the $\mathrm{X}$-axis components are assumed to be time-invariant and homogeneous, and the kinematics analysis is limited to Y-direction. Some denotations are defined as follows: $M_{m}$ mass of the slide of the master linear motor
$M_{s}$ mass of the slide of the slave linear motor

$M_{b}$ mass of the X-axis components

$B_{m}$ viscosity coefficient of the slide of the master linear motor

$B_{S}$ viscosity coefficient of the slide of the slave linear motor

$x_{m}$ displacement of the slide of the master linear motor

$x_{s} \quad$ displacement of the slide of the slave linear motor

$x_{b} \quad$ displacement of $\mathrm{X}$-axis components

$u_{m}(t)$ torque voltage input of the master linear motor

$u_{s}(t)$ torque voltage input of the slave linear motor

$f_{c} \quad$ friction force

$f_{k}$ deformed force of the $\mathrm{X}$-axis components in Y-direction

$K \quad$ stiffness of the $\mathrm{X}$-axis components in Y-direction

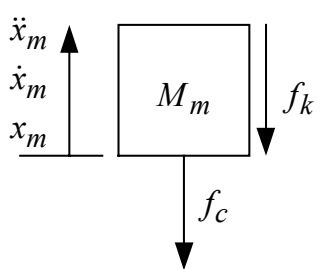

(a)

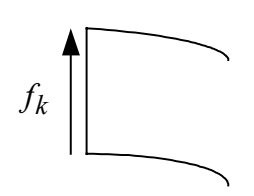

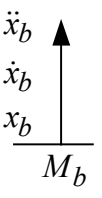

(c)

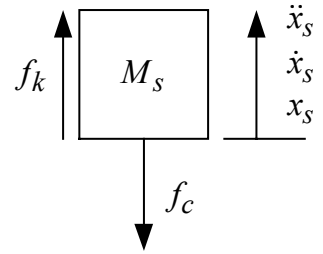

(b)

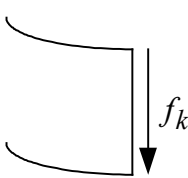

Fig. 3 Free body diagrams for (a) the slide of the master motor,(b) the slide of the slave motor and (c) the X-axis components

As seen in Fig. 3(a)(b)(c), for equilibrium of the force, the dynamic equations of the two slides are given respectively by

$$
\begin{aligned}
& M_{m} \frac{d^{2} x_{m}}{d t^{2}}+B_{m} \frac{d x_{m}}{d t}=u_{m}(t)-f_{c}-f_{k} \\
& M_{s} \frac{d^{2} x_{s}}{d t^{2}}+B_{s} \frac{d x_{s}}{d t}=u_{s}(t)-f_{c}+f_{k}
\end{aligned}
$$

Assuming that the material is homogeneous and linear, it is reasonable to simplify the estimated deformed force of the $\mathrm{X}$-axis components to be proportional to the synchronization error between the two motors, i.e.,

$$
f_{k} \approx K\left(x_{m}-x_{s}\right)
$$

where $x_{m}-x_{s}$ is the synchronization error.

From (1) and (2), the controlled plant can therefore be given as in Fig. 4. 


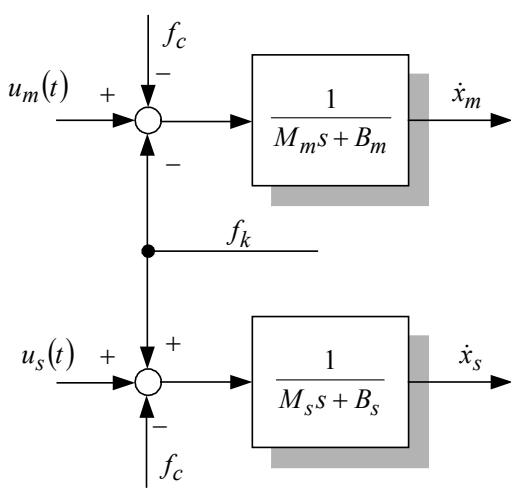

Fig. 4 The controlled plant of the parallel twin linear servomotor system

\section{Design of the Synchronous Control System}

General position control systems with a specific trajectory using S-curve planning are considered here. The designed parameters of the controllers are discussed and analyzed. Moreover, with repetitive motion signals input, the repetitive controller [14] is also incorporated to compensate the steady-state error, without disturbing the existent stable servo control system.

\subsection{General Position Control}

The proposed synchronous control system is shown in Fig. 5, where the gray box represents the controlled plant derived in Section II, as seen in Fig. 4. The control system is a MISO (Multi-Inputs and Single-Output) system, i.e., two inputs (the position command $x$ and the deformed force command $f^{*}$ ) and one output (the synchronization error $x_{m}-x_{s}$ ), where $C_{m v}$ and $C_{s v}$ are velocity-loop control gains of the master and slave motors respectively, $C_{m p}$ is the position-loop control gain of the master motor, and $C_{e}$ is the control gain for the deformed force. The master linear motor is driven by the high-stiffness position control and the slave linear motor is controlled in the velocity-loop following the motion of the master. For the requirements of the tracking performance and the decay rate of the synchronization error, the effect of the deformed force is compensated by the force controller $C_{e}$ and is estimated to be $K\left(x_{m}-x_{s}\right)$, as expressed in (3), where the corresponding stiffness $K=566 \mathrm{~N} / \mathrm{mm}$ of the X-axis components is experimentally determined. In fact, the desired deformed force command should be set zero to achieve zero synchronization error, i.e., $f^{*}=0$ and hence $x_{m}-x_{s}=0$. Here, the velocity-loop controllers $C_{m v}$ and $C_{S v}$ should be adjusted as PI control, and the position-loop controller $C_{m p}$ should be set as a proportional gain in practice for overshot-free design.

From the above description, it is known that the parameters of the controllers, $C_{m p}, C_{m v}$, and $C_{s v}$, are determined to guarantee the stability and the tracking performance. For position control, the proposed control system should be able to achieve a required tracking performance while the deformed force controller $C_{e}$ is adjusted as PI or proportional controllers. However, this may not be sufficient while the input signals are repetitive.
The repetitive control problem is detailed in the successive section.

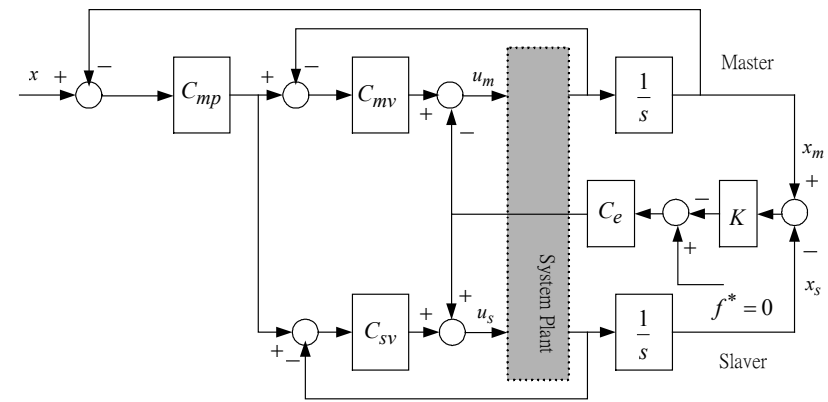

Fig. 5 The synchronous control system

\subsection{Repetitive Motion Control [14]}

Considering a periodic signal input with a period $T_{d}$, the repetitive controller $C_{r}$ is given as

$$
C_{r}(s)=\frac{s^{2}+2 \xi_{q} \omega_{q} s+\omega_{q}^{2}\left(1-e^{-\tau_{1} s}+e^{-\tau_{2} s}\right)}{s^{2}+2 \xi_{q} \omega_{q} s+\omega_{q}^{2}\left(1-e^{-\tau_{1} s}\right)}
$$

where $\xi_{q}=0.707, \tau_{1}=T_{d}-\tau_{q}$, and $\tau_{2}=T_{d}-\tau_{q}-\tau_{b}$.

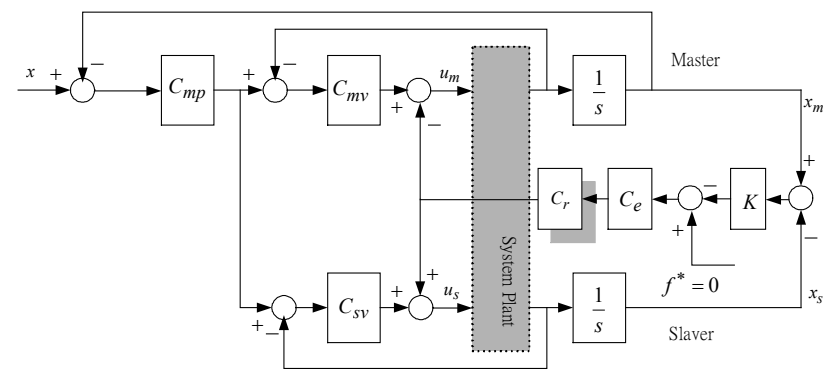

Fig. 6 Synchronous control system with the controller $C_{r}$ defined in (4)

From the repetitive control theory [14], the designed parameters, i.e., $\omega_{q}, \tau_{q}$, and $\tau_{b}$, are determined by the frequency domain specifications of the original stable control system without the repetitive control action. Considering the control system shown in Fig. 6, the following transfer function, where $x_{s}$ is the output, and $x_{m}$ and $x$ are the two inputs, is derived and analyzed.

$$
x_{s}=\frac{A_{2}(s)}{A_{1}(s)} x_{m}+\frac{A_{3}(s)}{A_{1}(s)} x
$$

where

$$
\begin{aligned}
& A_{1}(s)=1-\frac{K C_{e} G_{m}(s)}{1+\Delta_{1}(s)} \frac{1}{s}-\frac{K C_{e} G_{s}(s)}{1+\Delta_{2}(s)} \frac{1}{s} \\
& A_{2}(s)=1-\frac{K C_{e} G_{m}(s)}{1+\Delta_{1}(s)} \frac{1}{s}-\frac{K C_{e} G_{s}(s)}{1+\Delta_{2}(s)} \frac{1}{s}-\frac{C_{m p} C_{s v} G_{s}(s)}{1+\Delta_{2}(s)} \frac{1}{s} \\
& A_{3}(s)=\frac{C_{m p} C_{s v} G_{s}(s)}{1+\Delta_{2}(s)} \frac{1}{s}-\frac{C_{m p} C_{m v} G_{m}(s)}{1+\Delta_{1}(s)} \frac{1}{s} \\
& \Delta_{1}(s)=1+C_{m v} G_{m}(s)+C_{m p} C_{m v} G_{m}(s) / s \\
& \Delta_{2}(s)=1+C_{s v} G_{s}(s)
\end{aligned}
$$


According to the above analysis, the designed parameters of the repetitive controller are determined by the specifications of the frequency response of the system in (5).

\section{Experimental Studies}

Figure 7 shows the hardware setup for the experiments of the synchronous control scheme, as can be seen that the two parallel linear motors form the Y-axis. The machine working area is approximately $500 \mathrm{~mm} X 500 \mathrm{~mm}$. The specifications for the linear motors (LINERRSERV), manufactured by YOKOGAWA Ltd. of Japan, are listed in the Table 1. The drivers of the linear motors are all set to thrust mode for the present study. In the thrust mode, current flows through the motor according to the current command voltage $(-8$ to $+8 \mathrm{~V})$ from the high-level controller, and the motor output thrust depends on the current. Therefore, the thrust is 0 at $0 \mathrm{~V}$ of command voltage, and the maximum thrust $(100 \mathrm{~N})$ is produced at $8 \mathrm{~V}$. A TMS320C32 digital signal processor (DSP) is employed in the proposed synchronous control scheme for real-time operation.

\begin{tabular}{|c|c|}
\hline Max. force (N) & 100 \\
\hline Max. speed (m/s) & 0.87 \\
\hline Encoder resolution (um) & 0.5 \\
\hline Max. load (N) & 200 \\
\hline
\end{tabular}

Table 5.1 Specifications of the linear motor

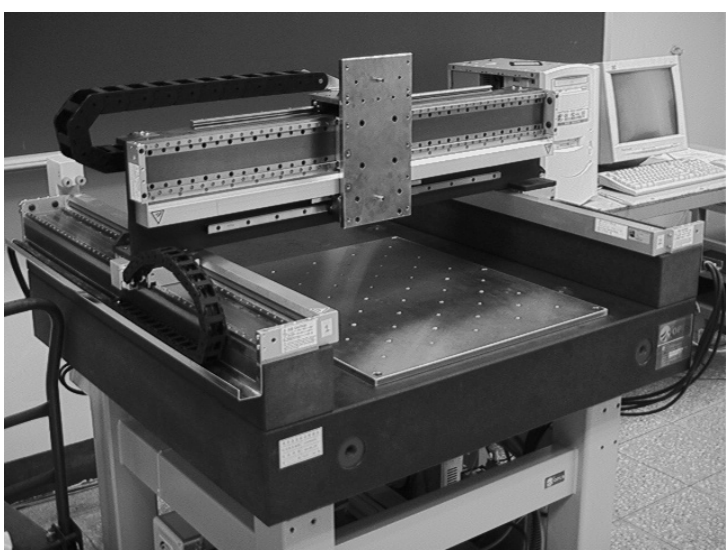

Fig. 7 The experimental setup

Without the control action, the transfer functions of the controlled plants (i.e., the three linear motors and the saddle) in the thrust mode are measured using a frequency spectrum analyzer.

For the master linear motor, the transfer function is

$$
G_{m}(\mathrm{~s})=\frac{12049.6}{s+88.2} \frac{\mathrm{mm} / \mathrm{s}}{\text { voltage }}
$$

$$
G_{S}(s)=\frac{10955.7}{s+85.9} \frac{\mathrm{mm} / \mathrm{s}}{\text { voltage }}
$$

For the linear motor of $\mathrm{X}$-axis,

$$
G_{x}(s)=\frac{12425.8}{s+89.9} \frac{\mathrm{mm} / \mathrm{s}}{\text { voltage }}
$$

From the measured transfer functions defined in (6) and (7), for the required tracking performance and stability, the corresponding controllers, $C_{m v}, C_{s v}, C_{m p}$, and $C_{e}$ of the control system in Fig. 5 are chosen as follows.

$$
\begin{aligned}
& C_{m v}(s)=0.75+0.1 / s \\
& C_{s v}(s)=0.91+0.2 / s \\
& C_{m p}(s)=0.8 \\
& C_{e}(s)=1.2
\end{aligned}
$$

According to the repetitive control theory [14], the controller $C_{r}$ of the control system in Fig. 6, is given by

$$
C_{r}(s)=\frac{s^{2}+195 s+19044\left(1-e^{-\tau_{1} s}+e^{-\tau_{2} s}\right)}{s^{2}+195 s+19044\left(1-e^{-\tau_{1} s}\right)}
$$

where $\tau_{1}=T_{d}-0.011$, and $\tau_{2}=T_{d}-0.021$.

Two experiments are considered, i.e., the general position control for the parallel linear servomotor system, and the repetitive motion control for the same servo system with repetitive controller. In the general control experiment, two desired trajectories with S-curve planning at low and high speeds respectively are considered. For the low speed case, the parameters for the command are the position $r=500 \mathrm{~mm}$, the maximum velocity

$v_{\max }=500 \mathrm{~mm} / \mathrm{s}$, the maximum acceleration

$a_{\max }=2800 \mathrm{~mm} / \mathrm{s}^{2}$, and the average acceleration

$a_{\text {ave }}=1600 \mathrm{~mm} / \mathrm{s}^{2}$, as shown in Fig. 8. The

synchronization error response of the parallel linear servomotor system is shown in Fig. 9. For the high-speed condition, the maximum velocity $v_{\max }$ is $870 \mathrm{~mm} / \mathrm{s}$, and other parameters remain unchanged, as shown in Fig. 10. The synchronization error response is shown in Fig. 11. As can be seen, the transient synchronization error of the low speed condition $(500 \mathrm{~mm} / \mathrm{s})$ is lower than that of the high speed condition $(870 \mathrm{~mm} / \mathrm{s})$. Moreover, for both cases the steady-state synchronization errors can reach $0.5 \mu \mathrm{m}$, which is the encoder resolution. Note that the synchronization error, as previously mentioned, is the error between the two parallel motors.

For the slave linear motor, 


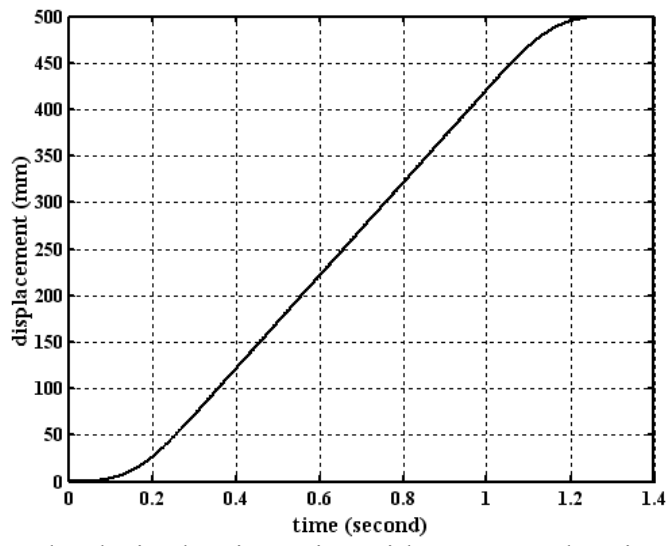

Fig. 8 The desired trajectories with S-curve planning at low speed condition

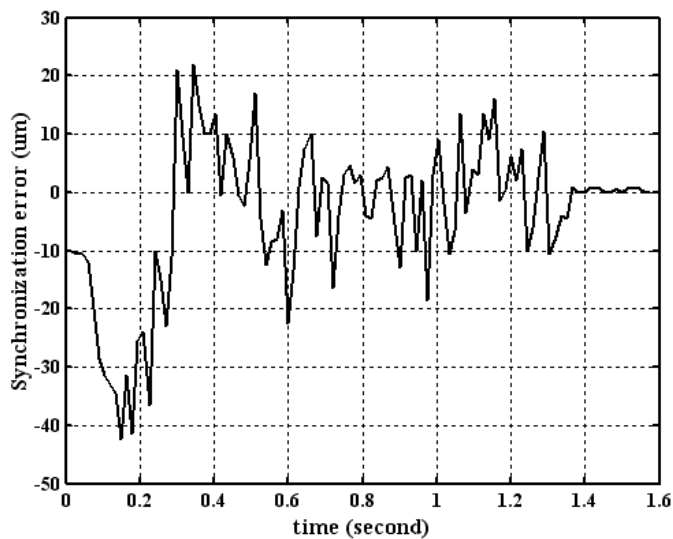

Fig. 9 Synchronization error response at low speed condition

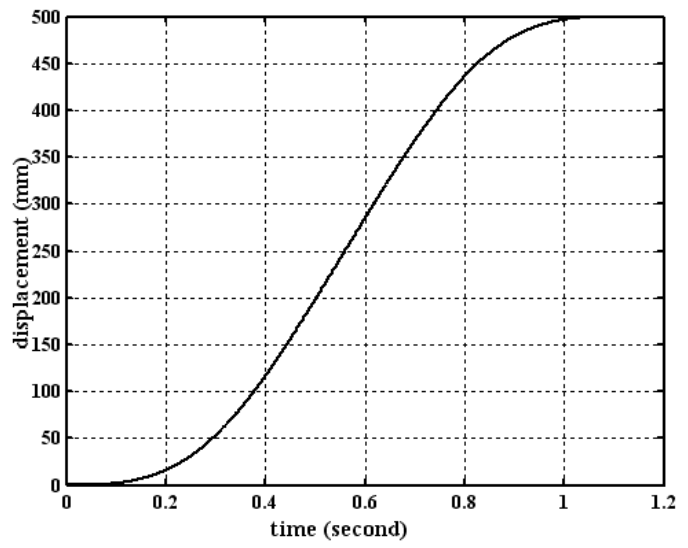

Fig. 10 The desired trajectories with S-curve planning at high speed condition

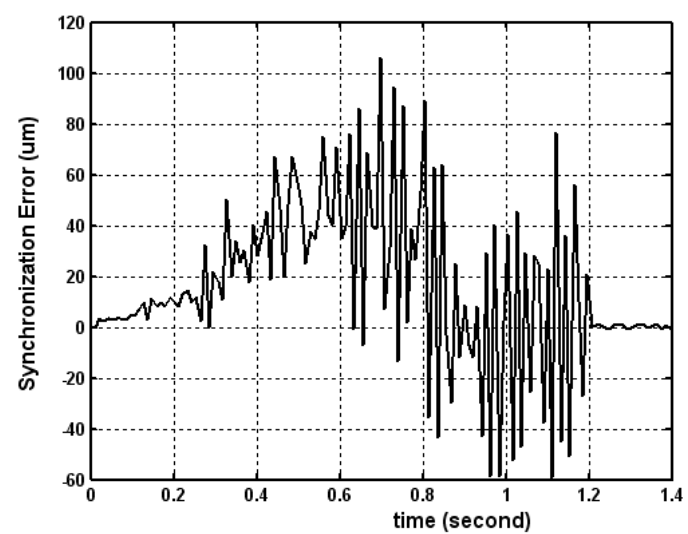

Fig. 11 Synchronization error response at high-speed condition
In the repetitive control experiment, the periodic motion command of $x=50 \sin (2 \pi t / 0.5)$ with a period $T_{d}=0.5$ is applied to both cases with and without repetitive control. The synchronous control system without repetitive control results in a sinusoidal error in the steady state as shown in Fig. 12. However, the tracking error of the control system with the repetitive controller decays rapidly within the first two cycles, as shown in Fig. 13. This demonstrates the effectiveness of the proposed repetitive controller incorporated in the synchronous control system for periodic motion.

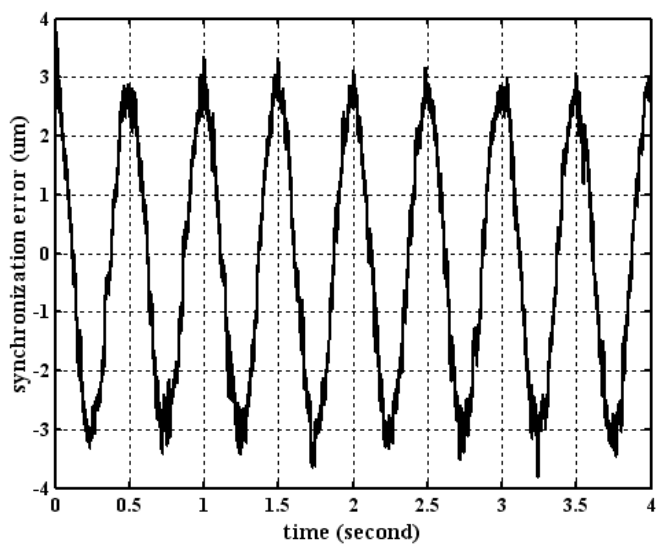

Fig. 12 Synchronization error response for periodic signal inputs without repetitive control

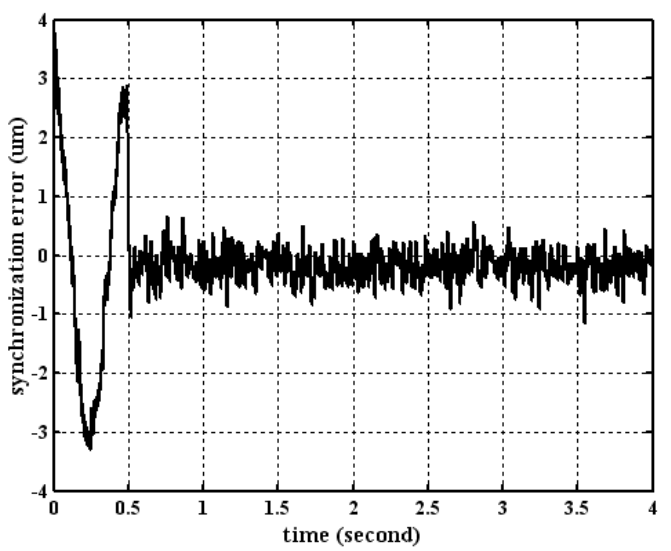

Fig. 13 Synchronization error response for periodic signal inputs with repetitive control

\section{Conclusions}

This paper has successfully developed a new synchronous control technique based on the master/slave control and repetitive control. Satisfactory tracking response for the servo system with a pair of parallel linear servomotors can be achieved within 1.5 seconds at the maximum speed of the motors $(870 \mathrm{~mm} / \mathrm{s})$ according to the experimental results. With the incorporation of a repetitive controller, the error caused by periodic signal inputs has effectively been reduced. It can be concluded that high speed machining can be achieved with the proposed synchronous control technique.

\section{Acknowledgements}

The work described in this paper has been carried out with the support of the National Science Council of Taiwan under the grant NSC 90-2212-E-006-094, for which the authors express their grateful thanks. 


\section{References}

[1] D. M. Alter and T. C. Tsao, "Implementation of a direct drive linear motor actuator for dynamic control of the turning process," Proceedings of American Automatic Control Conference, San Francisco, CA., June 1993.

[2] L. Feng, Y. Koren, and J. Broenstein, "Cross-coupling motion controller for mobile robots," IEEE Transactions on Control Systems, pp. 35-43, December 1993.

[3] H. Haack and M. Tomizuka, "Zero phase error tracking algorithm for digital control," ASME Journal of Dynamic Systems, Measurement, and Control, vol. 113, pp. 6-10, 1991.

[4] W. C. Johnson, K. Srinivasan, and P. Kulkarni, "Digital control algorithms for electromechanical machine tool feed drives," Proceedings of the 12th North American Manufacturing Research Conference, pp. 447-453, Houghton, Michigan, 1984.

[5] Y. Koren, "Cross-coupled biaxial computer controls for manufacturing systems," ASME Journal of Dynamic Systems, Measurement, and Control, vol. 102, pp. 265-272, 1980.

[6] P. K. Kulkarni and K. Srinivasan, "Cross-coupled control of bi-axial feed drive servomechanism," $A S M E$ Journal of Dynamic Systems, Measurement, and Control, vol.112, no. 2, 1990.

[7] G. M. Lammers, "Linears lead in ultrasmooth motion," Machine Design, pp.60-64, September 1994.

[8] A. E. Middleditch and F. W. Paul, "Dynamic performance of a computer numerical control system for multi-axis contouring machine tools," ASME Winter Annual Meeting, Detroit, Mich., Paper no. 73-WA/Aut-13, 1973.

[9] A. N. Poo, J. G. Bollinger, and G. W. Younkin, "Dynamic errors in type-1 contouring systems," IEEE Transactions on Industry Applications, vol. 1A-8, no. 4, pp. 471-484, 1972.

[10]P. Sarachik and J. R. Ragazzini, "A 2-dimensional feedback control system," Transactions of the AIEE, vol. 76, part II, pp. 55-61, 1957.

[11]D. Sun, H. N. Dong, and S. K. Tso, "Tracking stabilization of differential mobile robots using adaptive synchronization control," Proceedings of IEEE International Conference on Robotics and Automation, 2002.

[12]M. Tomizuka, J. S. Hu, and T. C. Chiu, "Synchronization of two motion control axes under adaptive feedforward control," ASME Journal of Dynamic Systems, Measurement, and Control, vol. 114, no. 6, pp. 196-689, 1992.

[13] M. Tomizuka, "Design of digital tracking controller for manufacturing applications," Proceedings of the Symposium on Control Methods for Manufacturing Processes, ASME Winter Annual Meeting, Chicago, III, DSC vol. 9, pp. 71-78, 1988.

[14] M. C. Tsai and W. S. Yao, "Design of a plug-in type repetitive controller for periodic inputs", IEEE Transactions On Control Systems Technology, vol. 10, no. 4, pp. 547-555, July 2002.

[15] L. F. Yang and W. H. Chang, " Synchronization of two-gyro precession under cross-coupled adaptive feedforward control," Journal of Guidance, Control, and Dynamics, vol. 19, no. 3, pp. 534-539, 1996. 